\title{
Perfil Socioeconômico e Farmacoterapêutico dos Usuários do Programa Farmácia Popular em Juazeiro do Norte, CE, Brasil
}

\author{
Socioeconomic and Pharmacotherapeutic Profile of the Users from \\ Popular Pharmacy Program in Juazeiro do Norte, CE, Brazil
}

Recebido em: 22/10/2016 Aceito em: $17 / 04 / 2017$
Emanuela Machado Silva SARAIVA ${ }^{1}$; Aron Paiva Mota FLORÊNCIO' ${ }^{1}$; Natália Bitu PINTO $^{1}$; Ricardo Peres do SOUTO ${ }^{2}$; Maria de Fátima Rocha BARRETO' Gustavo de Oliveira ALENCAR ${ }^{1}$; Camila Alencar BEZERRA ${ }^{1}$

${ }^{1}$ Faculdade de Juazeiro do Norte - FJN. Departamento de Farmácia. Rua São Francisco, 1224, Bairro São Miguel, CEP: 63010-475. Juazeiro do Norte, Ceará, Brasil.

${ }^{2}$ Faculdade de Medicina do ABC - FMABC. Departamento de Morfologia e Fisiologia. Avenida Príncipe de Gales, 821, Bairro Príncipe de Gales, CEP 09060-650. Santo André, São Paulo, Brasil.E-mail: emanuelams@hotmail.com

\section{ABSTRACT}

According to Brazilian Constitution, Health is a right of all population and should be provided by the State. Some public policies used to and continued to be adopted with the intention of elevating the quality of the health services and enlarging the access of the population to the essential medications; to an example of those policies, the federal government instituted in 2004 the Popular Pharmacy Program of Brazil (PPPB). The present article has as objective describing the socioeconomic and pharmacotherapeutic profile of the users of the Popular Pharmacy Program attended in the Juazeiro do Norte's unity in the region of Ceará. It is an exploratory, descriptive study, with the quantitative approach with 100 interviewees of both sexes sample, between 20 and 70 years old, being the collection of data accomplished through a semi-structured questionnaire. Among the 100 participants of the research, 77\% were female. $22 \%$ of the interviewees aged $61-70$ years; $79 \%$ affirmed to be no literateness; $78 \%$ claimed to have $1-2$ minimum wages income; $22 \%$ of the respondents were hypertensive; $61 \%$ said the medicines they used were for hypertension control; $96 \%$ affirmed that PFPB met their expectations, and 69\% declared having no conditions to finance the medication treatment. Besides the perspective of public health, the PFPB plays an important social role of the public compromises with to the disadvantaged Brazilian citizens. Amid the current discussions about the PFPB effectiveness and viability, it was possible to suggest strategies allowing to improve the program, such as the revision of the medicines list and the inclusion of more drugs, as well to enlarge the PFPB popularization.

Keywords: Essential Medicines. National Policy of Medicines. Public Policy of Health.

\section{RESUMO}

De acordo com a Constituição Brasileira, Saúde é um direito de todos e dever do Estado. Algumas políticas foram e continuam sendo adotadas com o intuito de elevar a qualidade dos serviços de saúde e alargar o acesso da população aos medicamentos essenciais, a exemplo dessas políticas o governo federal instituiu em 2004, o Programa Farmácia Popular do Brasil (PFPB). O presente artigo tem como objetivo traçar o perfil socioeconômico e farmacoterapêutico dos usuários do Programa Farmácia Popular atendidos na unidade de Juazeiro do Norte-CE. Trata-se de um estudo exploratório, descritivo, com abordagem quantitativa com amostra de 100 entrevistados de ambos os sexos, entre 20 e 70 anos, sendo a coleta de dados realizada através de questionário semiestruturado. Dentre os 100 participantes da 
pesquisa, $77 \%$ eram do sexo feminino; $22 \%$ têm entre $61-70$ anos de idade; $79 \%$ declaram-se não alfabetizado; $78 \%$ afirma ter renda familiar entre $1-2$ salários mínimos; $22 \%$ dos entrevistados eram hipertensos; $61 \%$ dos medicamentos destinam-se ao tratamento da hipertensão; $96 \%$ afirmam que o PFPB atende suas expectativas; $69 \%$ declara não ter condições de custear o tratamento medicamentoso. Além da perspectiva de saúde pública, o PFPB, desempenha um papel social importante de responsabilidade pública para com a população carente efetividade e viabilidade desse programa, demonstra-se o perfil do seu usuário, ao passo que se sugere estratégias que permitam um maior alcance do programa, tais como a revisão do elenco de medicamentos para inclusão de mais fármacos e maior divulgação.

Palavras-Chave: Medicamentos Essenciais. Política Nacional de Medicamentos. Políticas Públicas de Saúde.

\section{INTRODUÇÃO}

É constitucional que o cidadão seja amparado pelo Estado, por meio de programas de promoção, proteção e recuperação da saúde. De acordo com o artigo 196 da Constituição, "A saúde é direito de todos e dever do Estado, garantido mediante políticas sociais e econômicas que visem à redução do risco de doença e de outros agravos e ao acesso universal e igualitário às ações e serviços para sua promoção, proteção e recuperação" (1).

De acordo com a Constituição Federal Brasileira, por meio da Lei Orgânica de Saúde nº 8.080/90, que regulamenta o Sistema Único de Saúde (SUS) e estabelece a organização básica das ações e serviços em seu contexto (2), é assegurado o provimento da assistência terapêutica integral em que está inserida a assistência farmacêutica.

O Ministério de Saúde (MS) na sua Resolução de $n^{\circ} 338 / 04$, aprovou a Política Nacional de Assistência Farmacêutica (PNAF) a qual complementa a Política Nacional de Saúde e garante os princípios da equidade, universalidade e integralidade, com ações voltadas à proteção, promoção e recuperação da saúde, enfatizando o medicamento como insumo essencial que ressalta no seu uso racional (3). A Assistência Farmacêutica (AF) é uma estratégia da atenção básica que contribui para efetivar as ações do SUS e leva ao aumento da qualidade de vida da população, garantindo seu acesso aos medicamentos estabelecidos como essenciais, portanto, assegura o direito dos indivíduos à saúde (4).

O Programa Farmácia Popular do Brasil (PFPB) foi instituído por meio da Lei ${ }^{\circ} 10.858 / 2004$, que autorizou a Fundação Oswaldo Cruz (FIOCRUZ) a disponibilizar e dispensar medicamentos mediante ressarcimento, e pelo Decreto $\mathrm{n}^{\circ} 5.090 / 2004$, que regulamenta a Lei 10.858 e estabelece o Programa Farmácia Popular do Brasil. Segundo o Ministério da Saúde (2004), é uma iniciativa do Governo Federal e tem como objetivo ampliar o acesso aos medicamentos considerados essenciais para a população, com intuito de sempre atingir uma parcela que não possui rendimento suficiente para o tratamento medicamentoso $(5,6,7)$.

O referido Programa sofreu alterações por meio da Portaria No 111, 28 de Janeiro de 2016, que dispõe sobre o Programa Farmácia Popular do Brasil e que consiste na disponibilização de medicamentos e/ou correlatos à população, pelo Ministério da Saúde através dos seguintes meios: "Rede Própria", constituída por Farmácias Populares, em parceria com os Estados, Distrito Federal e Municípios (8).

Em 2006, por meio da Portaria no 491, o Ministério da Saúde expandiu o Programa Farmácia Popular do Brasil, chamado "Aqui Tem Farmácia Popular”, mediante o credenciamento da rede privada de farmácias e drogarias, os quais são dispensados pelo seu valor de custo, representando uma redução de até $90 \%$ do valor de mercado (9).

Além dos medicamentos gratuitos para hipertensão, diabetes e asma, o Programa oferece à sociedade o tratamento de dislipidemia, rinite, Parkinson, osteoporose, glaucoma e antidepressivos, além de contraceptivos e fraldas geriátricas para incontinência. A aquisição desses insumos através do Programa requer a apresentação de documentos do paciente, dentre os quais o CPF e um documento com foto, juntamente com uma receita médica ou odontológica (9).

O presente estudo teve como objetivo traçar o perfil socioeconômico e farmacoterapêutico dos usuários do Programa Farmácia Popular atendidos na unidade de Juazeiro do Norte-CE.

\section{MÉTODO}

Foi realizado um estudo de caráter descritivo, com abordagem quantitativa, tomando-se como lócus a unidade de Farmácia Popular do município de Juazeiro do Norte, CE, sendo esta classificada pela Fiocruz como Farmácia Popular Rede Própria modelo II. 
A coleta de dados ocorreu durante os meses de Agosto e Setembro de 2015, em uma sala reservada no âmbito da própria farmácia através de uma entrevista semiestruturada aplicada pelo pesquisador.

A população utilizada para a realização deste estudo foi composta pelos usuários da unidade do Programa Farmácia Popular, da cidade de Juazeiro do Norte, CE, tendo sido abordados 100 pacientes no ato do atendimento na unidade, dos quais todos aceitaram participar voluntariamente da pesquisa, sendo estes indivíduos de ambos os sexos e com idade a partir de 20 anos. Foram excluídos da pesquisa os usuários que recebiam medicamentos por meio de procuração à terceiro.

O instrumento de coleta utilizado para a realização da pesquisa caracterizou-se como uma entrevista semiestruturada, com questões abertas e fechadas que abordavam assuntos relacionados aos aspectos socioeconômicos e de saúde, relativos ao Programa Farmácia Popular.

Na cidade de Juazeiro do Norte, o programa Farmácia Popular integra a rede de assistência farmacêutica que disponibiliza medicamentos da atenção básica à saúde. De acordo com consulta ao banco de dados da referida farmácia, eram atendidos em média 600 pacientes mensalmente. Dessa maneira, foi proposto abordar uma amostra de 1/6 dessa população, ou seja, aproximadamente $17 \%$ da demanda média mensal da unidade. O referido quantitativo representa uma amostragem não probabilística do tipo por conveniência.
A presente pesquisa obedeceu aos preceitos éticos conforme a Resolução CNS/MS N466/2012 que trata de pesquisa envolvendo humanos. Para tanto foi submetida à Plataforma Brasil, apreciada pelo Comitê de Ética em pesquisa da Faculdade de Juazeiro do Norte-CE e aprovada sob o Parecer de $\mathrm{n}^{\circ}$ 1.393.497.

\section{RESULTADOS E DISCUSSÃO}

Estima-se que a população municipal, no período da pesquisa, ultrapassava os 268.000 habitantes, sendo a cidade caracterizada por uma forte influência religiosa e econômica ao sul do estado do Ceará.

De maneira aleatória, foram entrevistados 100 pacientes, quecompareceram à sede da Farmácia Popular do Brasil (FPB) da cidade de Juazeiro do Norte, CE, para adquirir seus medicamentos. De acordo com o levantamento dos dados sociodemográficos foi possível identificar e traçar o perfil do usuário da referida unidade conforme apresentado na Tabela 1.

Foi obtido um percentual de $77 \%$ dos usuários do sexo feminino e $23 \%$ do sexo masculino. O mesmo ocorreu com os resultados obtidos por Takemoto e cols (2015), onde dos usuários, do Programa "Aqui tem Farmácia Popular", duzentos e dois eram do sexo feminino $(60,3 \%)$ e cento e trinta e três $(39,7 \%)$, do sexo masculino (10).

Tabela 1. Indicadores sociodemográficos que configuram o perfil dos usuários da Farmácia Popular do Brasil (FPB) da cidade de Juazeiro do Norte, CE (2015)

\begin{tabular}{|c|c|c|c|}
\hline Variáveis & & $N^{0}(n=100)$ & $\%$ \\
\hline Gênero & $\begin{array}{l}\text { Feminino } \\
\text { Masculino }\end{array}$ & $\begin{array}{l}77 \\
23\end{array}$ & $\begin{array}{l}77 \% \\
23 \%\end{array}$ \\
\hline Faixa etária & $\begin{array}{l}20 \text { a } 30 \text { anos } \\
31 \text { a } 40 \text { anos } \\
41 \text { a } 50 \text { anos } \\
51 \text { a } 60 \text { anos } \\
61 \text { a } 70 \text { anos } \\
\text { Acima de } 70 \text { anos }\end{array}$ & $\begin{array}{l}12 \\
17 \\
21 \\
17 \\
22 \\
11\end{array}$ & $\begin{array}{l}12 \% \\
17 \% \\
21 \% \\
17 \% \\
22 \% \\
11 \%\end{array}$ \\
\hline Estado Civil & $\begin{array}{l}\text { Casado } \\
\text { Solteiro } \\
\text { Viúvo } \\
\text { Separado }\end{array}$ & $\begin{array}{l}52 \\
21 \\
14 \\
13\end{array}$ & $\begin{array}{l}52 \% \\
21 \% \\
14 \% \\
13 \%\end{array}$ \\
\hline Tipo de moradia & $\begin{array}{l}\text { Própria } \\
\text { Alugada } \\
\text { Cedida }\end{array}$ & $\begin{array}{c}41 \\
53 \\
6\end{array}$ & $\begin{array}{c}41 \% \\
53 \% \\
6 \%\end{array}$ \\
\hline Escolaridade & $\begin{array}{l}\text { Alfabetizado } \\
\text { Não alfabetizado }\end{array}$ & $\begin{array}{l}21 \\
79\end{array}$ & $\begin{array}{l}21 \% \\
79 \%\end{array}$ \\
\hline Renda mensal do domicílio & $\begin{array}{l}1 \text { a } 2 \text { salários mínimos } \\
3 \text { a } 4 \text { salários mínimos } \\
5 \text { ou mais salários }\end{array}$ & $\begin{array}{l}78 \\
12 \\
10\end{array}$ & $\begin{array}{l}78 \% \\
12 \% \\
10 \%\end{array}$ \\
\hline
\end{tabular}


Foi observado gradiente positivo, com aumento da prevalência do conhecimento sobre o Programa até os 69 anos, com uma insignificante diminuição estatística nas idades mais avançadas. Com o aumento da idade e correspondente aumento da prevalência das doenças crônicas, a frequência na demanda aos serviços de saúde e do interesse pelas questões de saúde e de medicamentos apresentam tendências ao crescimento. Por conseguinte, o declínio da prevalência do conhecimento - sem significância estatística - a respeito do Programa Farmácia Popular do Brasil, observado entre os mais idosos, em que com o aumento do percentual de indivíduos que, com a progressão da idade, veem sua autonomia gradativamente comprometida, passam a serem cuidados por outros familiares ou cuidadores (11).

A quantidade de pacientes idosos atendidos pela unidade de FPB/Juazeiro do Norte, CE decorre da prevalência das doenças crônicas, visto que, com o avançar da idade vem consigo possíveis complicações de saúde, fazendo com que se tenha uma maior utilização de serviços de saúde, elevado consumo de medicamentos e aumento do número de hospitalizações (12).

A procura de medicamentos por pacientes idosos é considerada alta, pelo fato da maioria destes utilizarem medicamentos de uso contínuo devido à presença de doenças. Outro fator seria a disponibilidade em caráter gratuito para o tratamento de hipertensão e diabetes. Vale ressaltar também, que pacientes de meia idade
(41-50 anos) e até adultos jovens ( $\leq 40$ anos) também já apresentam doenças crônicas e já realizam o tratamento medicamentoso como ofertado pelo Programa Farmácia Popular.

$\mathrm{O}$ acesso aos medicamentos para os entrevistados era feito exclusivamente por meio do Programa Farmácia Popular ou rede de farmácias privadas credenciadas ao Programa que apresentava $86 \%$ dos entrevistados, quando os medicamentos prescritos compuserem a relação disponibilizada pelo Programa.

Dentre as doenças relatadas, $61 \%$ dos entrevistados afirmaram diagnóstico médico de Hipertensão e $22 \%$ de Diabetes mellitus, sendo estas e as demais doenças declaradas, mostradas na Figura 1.

O acesso, no Brasil, ao tratamento medicamentoso para a hipertensão é de $94 \%$. O maior acesso dessa distribuição está nas Regiões Sul e Sudeste relatada por Mengue et al., 2016. Em relação ao diabetes, o acesso, no Brasil, ao tratamento medicamentoso é de $95 \%$. O maior acesso dessa distribuição está na região Sudeste, com $97 \%$, e o menor na região Nordeste, com 93\% (13).

No Brasil, em média $72 \%$ das mortes são atribuídas às Doenças Crônicas Não Transmissíveis (DCNT). As principais causas de óbitos entre idosos são as doenças cardiovasculares $(36,0 \%)$, neoplasias $(14,7 \%)$, e as doenças do aparelho respiratório (12,6\%), as quais correspondem a mais de $60 \%$ dos óbitos, e a hipertensão

\section{DOENÇAS DECLARADAS PELOS ENTREVISTADOS}

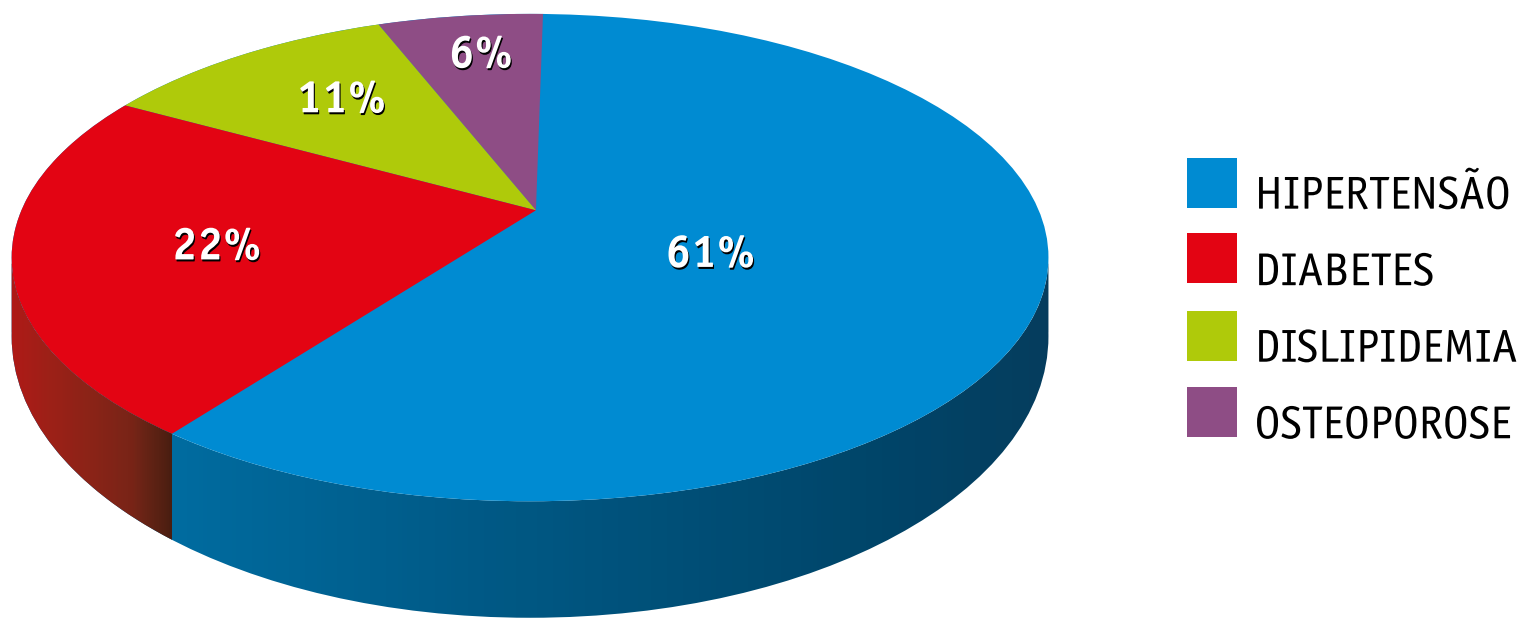

Figura 1. Agravos de saúde relatados pelos usuários entrevistados na Farmácia Popular do Brasil (FPB) da cidade de Juazeiro do Norte, CE (2015) 
(HAS) e o diabetes (DM) estão entre os principais fatores de risco para as doenças cardiovasculares. A HAS chega a acometer mais de $50 \%$ dos idosos e o DM atinge $20 \%$ deles. Com o avanço da idade, essas prevalências tendem a aumentar, havendo $50 \%$ de possibilidade das duas doenças estarem associadas. As doenças crônicas também constituem uma das principais causas de internações hospitalares, ressaltando a necessidade do diagnóstico precoce, da assistência médica e do acesso ao tratamento prescrito, sendo a insuficiência cardíaca a causa mais frequente de internação entre os idosos, das quais $12,1 \%$ para mulheres e $14,7 \%$ para os homens. Diabetes e hipertensão estão entre as seis principais causas somente entre as mulheres, e pneumonia, bronquite e acidente vascular cerebral entre as seis mais importantes causas para ambos os sexos (12).

Com relação ao tempo de diagnóstico da doença, a maioria dos entrevistados respondeu ter diagnosticado a doença crônica entre 5 a 10 anos antecedentes e 7\% afirmaram ter recebido o diagnóstico por menos de 1 ano, conforme mostra a Figura 2.

Pode ser observada uma total relação entre os medicamentos utilizados pelos entrevistados e as doenças por eles declaradas; dessa forma os medicamentos mais procurados na FPB de Juazeiro do Norte, CE destinam-se ao tratamento da hipertensão (61\%), dentre os quais se destacam a hidroclorotiazida, captopril, cloridrato de pro- pranolol, maleato de enalapril, losartana potássica e atenolol e $22 \%$ é destinado ao tratamento da diabetes, sendo disponibilizado para tal finalidade terapêutica o cloridrato de metformina e a glibenclamida; $11 \%$ visava o tratamento da dislipidemia, por meio da sinvastatina; e $6 \%$ à osteoporose, cujo medicamento ofertado pela FPB era o alendronato de sódio, conforme apresentado na Tabela 2.

Tabela 2. Medicamentos prescritos para tratamento das doenças relatadas pelos usuários entrevistados na Farmácia Popular do Brasil (FPB) da cidade de Juazeiro do Norte, CE (2015)

\begin{tabular}{|c|c|c|}
\hline Medicamentos & $\mathbf{N}$ & $\%$ \\
\hline \multicolumn{3}{|l|}{ Terapia Anti-hipertensiva } \\
\hline Atenolol $25 \mathrm{mg}$ & 09 & \multirow{6}{*}{61} \\
\hline Captopril 25mg & 09 & \\
\hline Cloridrato de Propranolol 40mg & 12 & \\
\hline Hidroclorotiazida $25 \mathrm{mg}$ & 53 & \\
\hline Maleato de enalapril $10 \mathrm{mg}$ & 05 & \\
\hline Losartana potássica $50 \mathrm{mg}$ & 44 & \\
\hline \multicolumn{3}{|l|}{ Terapia Hipoglicemiante } \\
\hline Cloridrato de metformina $500 \mathrm{mg}$ & 22 & \multirow{2}{*}{22} \\
\hline Glibenclamida 5mg & 18 & \\
\hline \multicolumn{3}{|l|}{ Terapia Hipolipemiante } \\
\hline Sinvastatina $20 \mathrm{mg}$ & 11 & 11 \\
\hline \multicolumn{3}{|l|}{ Terapia para 0steoporose } \\
\hline Alendronato de sódio $70 \mathrm{mg}$ & 06 & 6 \\
\hline
\end{tabular}

\section{TEMPO DE DIAGNÓSTICO}

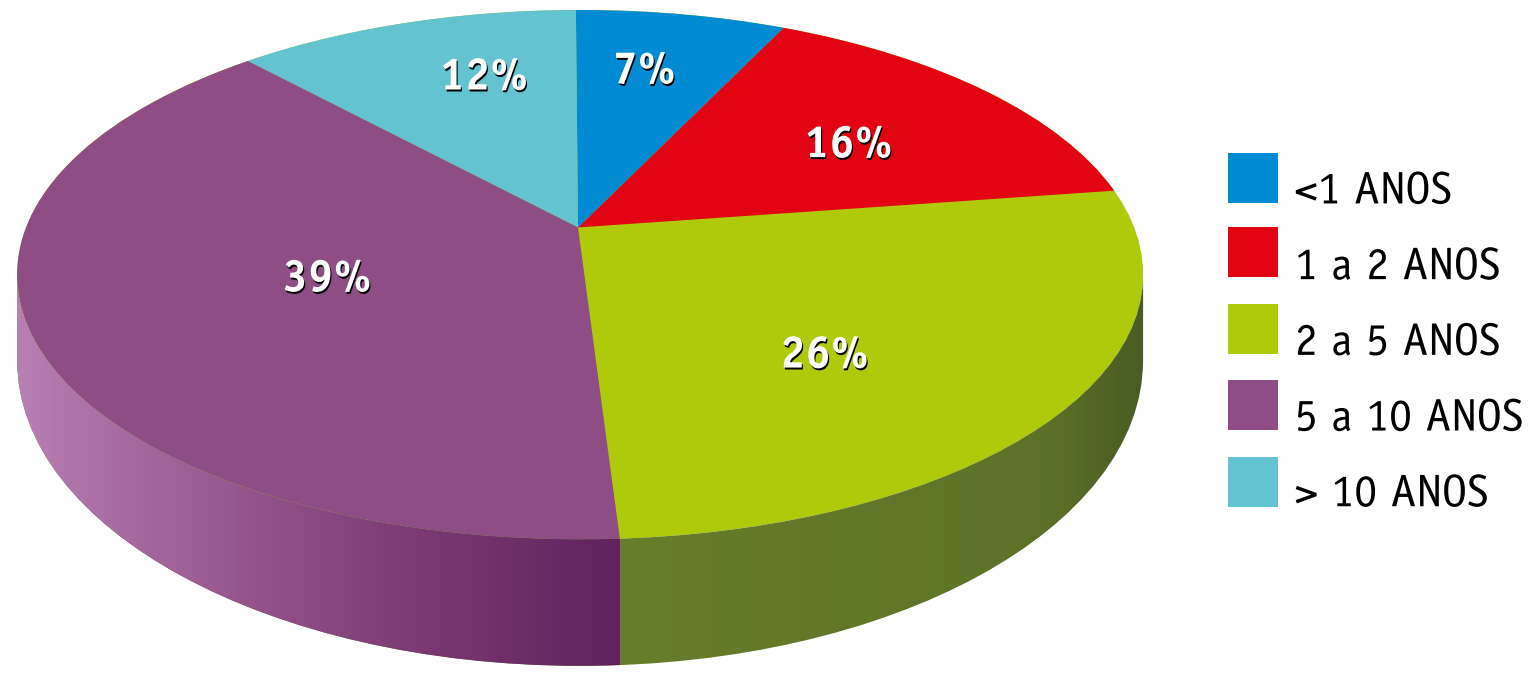

Figura 2. Tempo de diagnóstico das doenças relatadas pelos usuários entrevistados da Farmácia Popular do Brasil (FPB) da cidade de Juazeiro do Norte, CE (2015) 


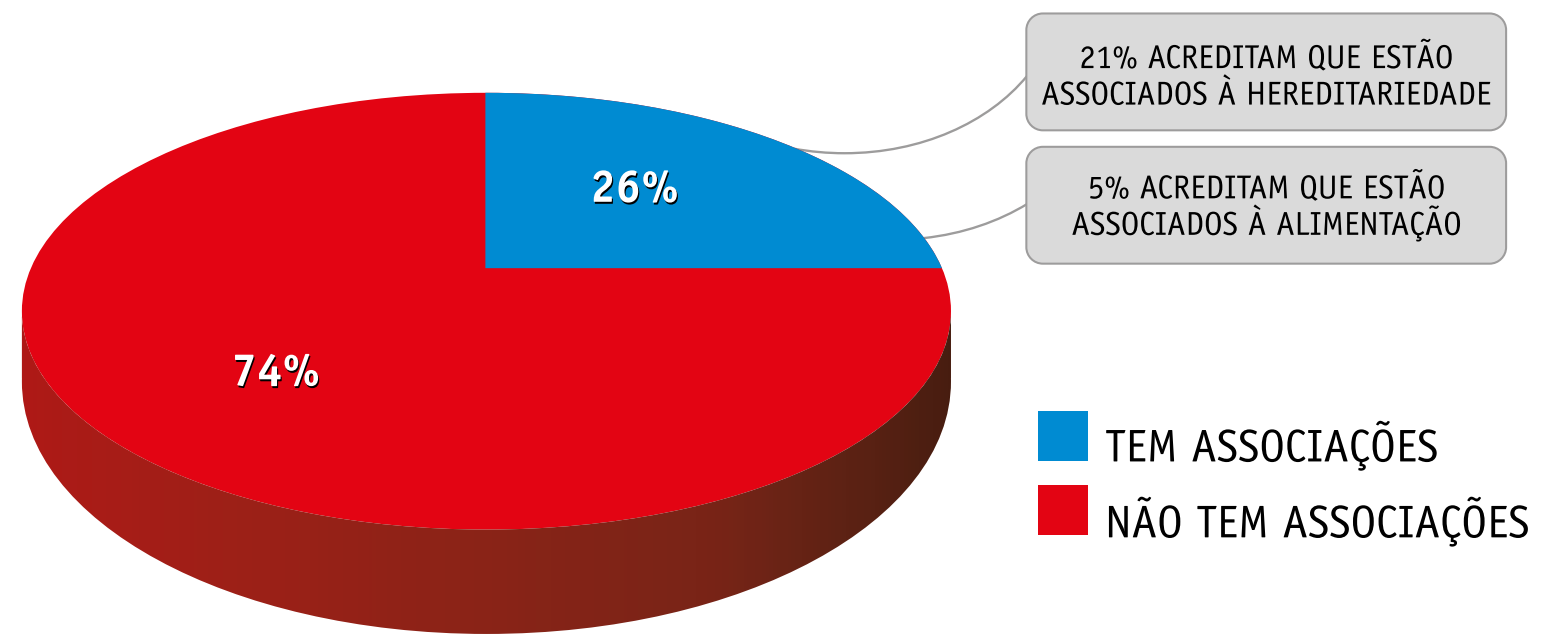

Figura 3. Fatores associados ao diagnóstico das doenças relatadas, de acordo com os usuários entrevistados na Farmácia Popular do Brasil (FPB) da cidade de Juazeiro do Norte, CE (2015)

Amorim e cols (2015), afirmaram que o medicamento mais dispensado pelo Programa Farmácia Popular do Brasil em uma drogaria de São Luís de Montes Belos, GO foi a losartana potássica, representando $40,80 \%$ do total de medicamentos dispensados pela farmácia (14).

No período de setembro de 2013 a janeiro de 2014, a Pesquisa Nacional sobre Acesso, Utilização e Promoção do Uso Racional de Medicamentos (PNAUM), considerou, além do diagnóstico referido, a indicação médica para o tratamento medicamentoso, segundo a qual foi verificado um maior uso de medicamentos para essas doenças: 7,1\% dos hipertensos não tinham indicação de tratamento com medicamentos; e daqueles que tinham indicação de tratamento com medicamentos, $6,0 \%$ não seguiam a terapêutica indicada. Para o diabetes, esses valores foram de $14,5 \%$ e $7,2 \%$ respectivamente. Entre pacientes com hipertensão e diabetes as frequências mais reduzidas de tratamento medicamentoso ou não utilização de medicamentos podem estar associadas a barreiras no acesso e na adesão ao tratamento farmacológico para o controle das doenças e as medidas de promoção da saúde como mudanças de hábitos e de estilo de vida (15).

O completo acesso aos medicamentos para hipertensão pode ser considerado a primeira etapa no tratamento dessa condição clínica, mas não o único. Em média $50,0 \%$ dos pacientes tratados não têm controle adequado da pressão arterial. Além da obtenção dos medicamentos, são necessários boa adesão ao tratamento, adequado manejo desses medicamentos e controle dos demais fatores que podem dificultar o controle da pressão arterial, tais como a elevada ingestão de sal ou a apneia obstrutiva do sono (16).

Sobre a associação de fatores intrínsecos e extrínsecos à condição da doença, $26 \%$ dos entrevistados presumiram que algum fator pode ter associação com o desencadeamento da condição patológica. Dentre esses, $21 \%$ acreditavam que questões de hereditariedade favorecem o surgimento da doença; e, 5\% afirmaram que os hábitos alimentares podem provocar a doença, como mostra a Figura 3.

Para 96\% dos entrevistados, a FPB de Juazeiro do Norte, CE atendia suas expectativas, pois eram motivados pela possibilidade de receberem, gratuitamente, os medicamentos prescritos pelo médico. Dessa forma, não necessitariam de desembolsar qualquer valor para adquiri-los ou ficariam sem o medicamento por não terem condições financeiras para comprá-los. Dentre os que afirmaram que o Programa não atendia às suas expectativas, o motivo mencionado foi o elenco de medicamentos ser restrito, conforme a Figura 4.

Com relação à disponibilidade dos medicamentos na FPB lócus da pesquisa, a maioria dos entrevistados (92\%), afirmou que os medicamentos sempre estavam disponíveis no momento da procura e apenas $8 \%$ disseram que nem sempre os medicamentos estavam disponíveis, tendo alguns que custear os medicamentos na rede privada e outros esperavamm o mês seguinte 
para conseguir o medicamento por não terem condições de comprá-los.

A partir dos motivos pelos quais os entrevistados afirmaram utilizar o Programa FPB (Figura 5), 69\% relataram não ter condições de custear os medicamentos para o tratamento de suas doenças, e ficaria impossível de adquiri-los nas redes privadas; 9\% optaram por receber os medicamentos pelo Programa FPB por acreditar na obrigatoriedade do governo em prover o acesso aos medicamentos pela população; $3 \%$ recebiam esses medicamentos porque acreditavam que os medicamentos distribuídos são de boa qualidade; e 5\% devido à proximidade da farmácia em relação da residência ou do local de trabalho.

Para $81 \%$ dos entrevistados, o uso da FPB era anterior à gratuidade de alguns medicamentos. Apenas 19\% passaram a utilizar esse Programa de acesso aos medicamentos após à gratuidade dos medicamentos destinados ao tratamento de doenças crônicas, como diabetes e hipertensão, ocorrido em fevereiro de 2011. Pode ser inferido que o fator gratuidade promoveu a ampliação do acesso à terapia medicamentosa pela população brasileira.

Foi indagado aos entrevistados sobre a utilização da rede credenciada denominada "Aqui tem Farmácia Popular". Apenas $20 \%$ dos entrevistados afirmaram receber seus medicamentos por meio do convênio do Pro- grama Farmácia Popular com a iniciativa privada, sendo que a maioria $(80 \%)$ recorria à sede do programa FPB na cidade de Juazeiro do Norte, CE.

Segundo Inocencio e Vivo (2011), o Programa Aqui tem Farmácia Popular restringe o acesso universal aos medicamentos, ferindo inclusive, o princípio da universalidade e do atendimento à saúde, trazendo transtornos à população mais carente desprovida de recursos financeiros e, que por esta razão, não tem poder de compra para iniciar e concluir seu tratamento, aumentando ainda mais a exclusão social (17).

\section{CONCLUSÃO}

O estudo realizado pode caracterizar que a população usuária do Programa Farmácia Popular do Brasil na cidade de Juazeiro do Norte, CE, em sua maioria era do gênero feminino, com idade entre 61-70 anos, casado, não alfabetizado, com renda domiciliar de um a dois salários mínimos e residentes em imóvel alugado. Fato notório na pesquisa foi o consumo de medicamentos destinados ao tratamento de DCNT por pacientes jovens, fato este fortemente relacionado ao comportamento sedentário informado pelos entrevistados, pois o sedentarismo é considerado um dos fatores de risco para o desenvolvimento da hipertensão.

\section{ATENDIMENTO ÀS EXPECTATIVAS}

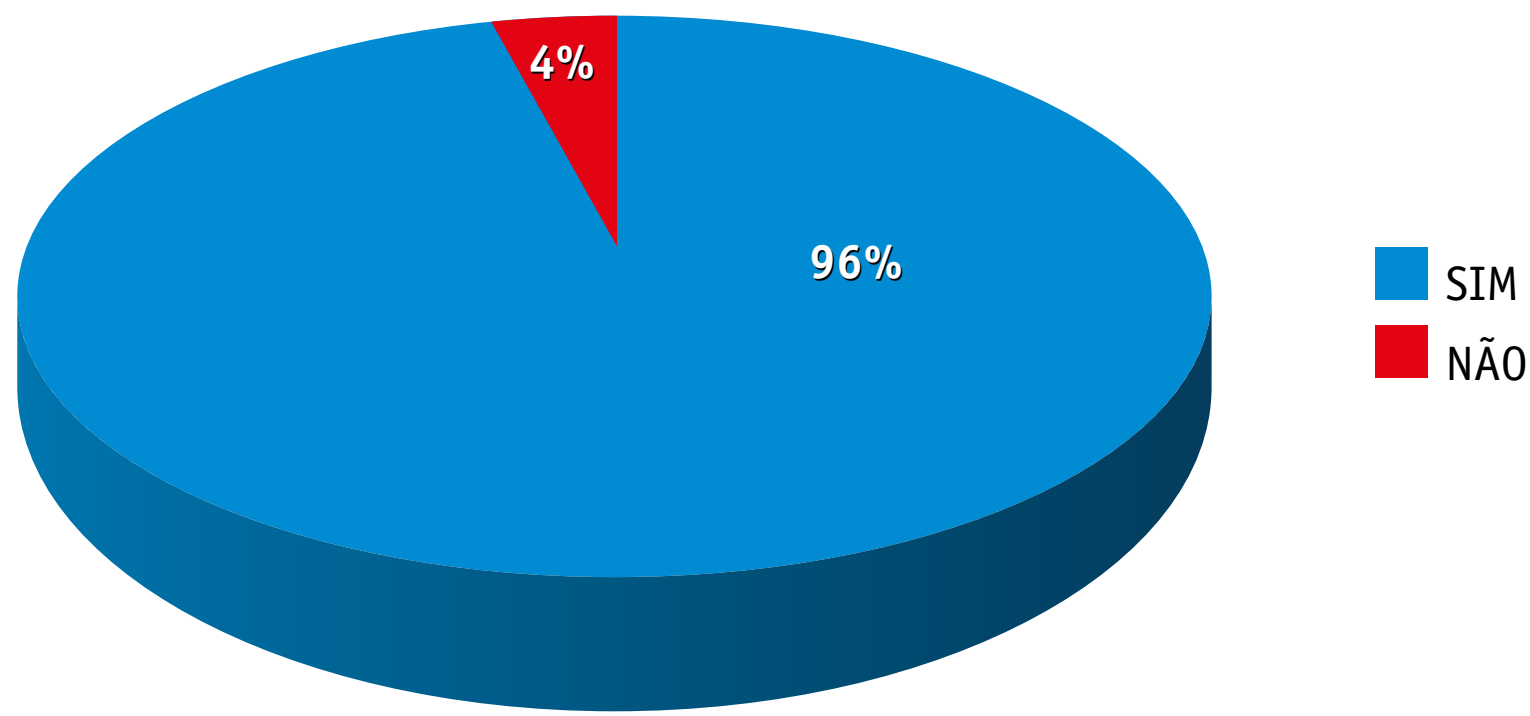

Figura 4. Atendimento às expectativas dos usuários entrevistados da Farmácia Popular do Brasil (FPB) da cidade de Juazeiro do Norte, CE (2015) 
As classes de medicamentos mais procuradas pelos entrevistados foram aquelas destinadas ao tratamento da hipertensão e diabetes.

Mais da metade dos entrevistados afirmou faltar condições financeiras para adquirir os medicamentos necessários ao tratamento da sua condição patológica. Diante desse fato, pode ser concluído que o Programa atingiu o objetivo de promover o acesso ao medicamento a uma considerável parcela da população brasileira. Contudo, além da perspectiva de saúde pública, o Programa Farmácia Popular do Brasil, unidade própria, de-

\section{REFERÊNCIAS}

1. Ministério da Saúde (BR). Constituição Federal. Artigos 196 a 200. Brasília (DF), 1988. Disponível: http://conselho.saude.gov.br/web_sus20anos/20anossus/legislacao/ constituicaofederal.pdf.

2. Ministério da Saúde (BR). Lei 8.080, de 19 de setembro de 1990. Dispõe sobre as condições para a promoção, proteção e recuperação da saúde, a organização e o funcionamento dos serviços correspondentes e dá outras providências. 1990. Brasília (DF). Conselho Nacional de Saúde. Disponível: http://conselho.saude.gov.br/legislacao/lei8080_190990.htm. Acesso em: 18 mar. 2015.

3. Ministério da Saúde (BR). Resolução No 338, de 06 de maio de 2004. Brasília (DF). Diário Oficial da União. Disponível em: http://bvsms.saude.gov.br/bvs/saudelegis/cns/2004/res0338_06_05_2004.html. Acesso em: 16 mar. 2015.

4. Portela AS, Leal AAF, Werner RPB, Simões MOS, Medeiros ACD. Políticas públicas de medicamentos: trajetória e desafios. São Paulo: Araraquara. Rev Ciênc Farm Básica Apl., 2010; 31(1):09-14.

5. Ministério da Saúde (BR). Lei No 10.858, de 13 de Abril de 2004. Autoriza a Fundação Oswaldo Cruz - Fiocruz a disponibilizar medicamentos, mediante ressarcimento, e dá outras providências. 2004. Brasília (DF). Diário Oficial da União. Disponível em: http://www.planalto.gov. br/ccivil_03/_ato2004-2006/2004/lei/110.858.htm. Acesso em: 22 abr. 2017.

6. Ministério da Saúde (BR). Decreto No 5.090, de 20 de Maio de 2004. Regulamenta a Lei no-10.858, de 13 de abril de 2004, e institui o programa "Farmácia Popular do Brasil", e dá outras providências. 2004. Brasília (DF). Diário Oficial da União. Disponível em: http://www. planalto.gov.br/ccivil_03/_ato2004-2006/2004/decreto/ d5090.htm. Acesso em: 25 fev. 2017.

7. Costa HS, Borba ERC, Vilanova CM, Costa DM, Coutinho DF. Programa Farmácia Popular do Brasil: evolução sempenha um papel social importante de responsabilidade pública para com a população carente do nosso país.

Em meio às atuais discussões sobre a efetividade e viabilidade desse Programa, foi possível mostrar o perfil do seu usuário e sugerir estratégias que permitam um maior alcance do programa, tais como a revisão do elenco de medicamentos para inclusão de mais fármacos a serem disponibilizados aos cidadãos brasileiros, bem como uma maior divulgação entre os profissionais de saúde e população em geral.

do Programa na cidade de São Luís - MA. Maranhão: São Luíz. Rev Pesq Saúde. 2010; 11(3): 16-19.

8. Ministério da Saúde (BR).Portaria No 111, de 28 de janeiro de 2016. Programa Farmácia Popular do Brasil (PFPB). 2016. Brasília (DF). Diário Oficial da União. Disponível em: http://bvsms.saude.gov.br/bvs/saudelegis/gm/2016/prt0111_28_01_2016.html. Acesso em: 21 abr. 2017.

9. Ministério da Saúde (BR). Portal da Saúde. 2015. Brasília (DF). Disponível em: http://portalsaude.saude.gov. br/index.php/o-ministerio/principal/leia-mais-o-ministerio/346-sctie-raiz/daf-raiz/farmacia-popular/11-farmacia-popular/18008-programa-farmacia-popular-do-brasil. Acesso em: 17 mar. 2015.

10. Takemoto DCS, Cruz GJF, Corsato RF, Miyamoto CA. Perfil do Usuário do Programa Farmácia Popular. Mato Grosso do Sul: Três Lagoas. Rev. Conexão Eletrônica, 2015. 12(1): 1-10.

11. Costa KS, Francisco PMSB, Barros MBA. Conhecimento e utilização do Programa Farmácia Popular do Brasil: Estudo de Base Populacional no Município de Campinas-SP. São Paulo: Campinas. Epidemiol. Serv. Saúde. 2014. 23(3): 397-408. DOI: 10.5123/S167949742014000300003.

12. Miranda VIA. Utilização do Programa Farmácia Popular entre Idosos da Zona Urbana de Pelotas/RS. (Trabalho de Mestrado). Universidade Federal de Pelotas, Rio Grande do Sul. 2014. Disponível em: http://www.epidemio-ufpel.org.br/uploads/teses/Dissertacao_posbanca.pdf.

13. Mengue SS, Bertoldi AD, Boing AC, NUL Tavares, Pizzol TSD, Oliveira MA, Arrais PSD, Ramos LR, Farias MR, Luiza VL, Bernal RTI, Barros AJD. Pesquisa Nacional sobre Acesso, Utilização e Promoção do Uso Racional de Medicamentos (PNAUM): Métodos do Inquérito Domiciliar. São Paulo. Rev. Saúde Publica. 2016. 50(supl 2): 4s. DOI:10.1590/S1518-8787.2016050006156. 


\section{Inotama}

14. Amorim MES, Ferreira MRR, Oliveira SAS, Fernandes CKC. Programa Farmácia Popular do Brasil em uma Drogaria de São Luís de Montes Belos - GO. Rev. Eletrônica FMB. 2015. 8(1): 1-13. Disponível em: http://revista.fmb.edu.br/index.php/fmb/article/viewFile/24/21. Acesso em: 26 mar. 2017.

15. Costa KS, Tavares NUL, Mengue SS, Pereira MA, Malta DC, Júnior JBS. Obtenção de medicamentos para hipertensão e diabetes no Programa Farmácia Popular do Brasil: Resultados da Pesquisa Nacional de Saúde, 2013. Brasília: Distrito Federal. Epidemiol. Serv. Saúde. 2016; 25(1): 33-44. DOI: 10.5123/S1679-49742016000100004.
16. Mengue SS, Bertoldi AD, Ramos LR, Farlas MR, Oliveira MA, Tavares NUL, Arrais PSD, Luiza VL, Pizzol TSD. Acesso e Uso de Medicamentos Para Hipertensão Arterial no Brasil. São Paulo. Rev Saúde Pública. 2016. 50(Suppl 3): 8s. DOI: 10.1590/S1518-8787.2016050006154.

17. Inocencio M, Vivo B. Acesso a Medicamentos: Análise das Estratégias do Estado para o Desenvolvimento do Programa Farmácia Popular. São Paulo. Cadernos Gestão Pública e Cidadania. 2011; 16(59): 201-221. DOI: http://dx.doi.org/10.12660/cgpc.v16n59.3700. 